\title{
Uso combinado de VBA y Solver de Excel para la realización de ejercicios de optimización en ficheros Excel fácilmente evaluables
}

\author{
Asunción Santafé Morosa, José M. Gozálvez Zafrilla ${ }^{a}$, Fidel Toldrá Reigb, David \\ Catalán Martínez ${ }^{\mathrm{b}}$, Manuel Cesar Martí Calatayud ${ }^{\mathrm{a}}$

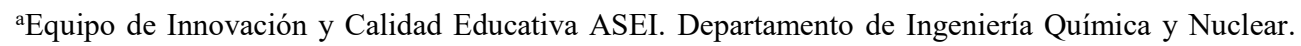 \\ Instituto de Seguridad Industrial y Medioambiental. E.T.S. Ingenieros Industriales de la Universitat \\ Politècnica de València, jmgz@iqn.upv.es, assanmo@iqn.upv.es bInstituto de Tecnología Química- \\ CSIC. Universitat Politècnica de València, dacamar3@itq.upv.es, fitolrei@itq.upv.es.
}

\begin{abstract}
The present study is focused on the exploitation of the potential of VBA and Solver tools of Excel during the execution of a computer practice lesson within the subject of "Analysis and simulation of processes" corresponding to the bachelor's degree of Chemical Engineering. VBA was used to design an Excel spreadsheet that integrates the following items: the assignment of different series of experimental data to the students, the guided and structured execution of three exercises, and the automated evaluation of the practice questions by the professor. The integrated and structured design of the lesson has advantages for both the students and the professors, since it simplifies the execution and evaluation of the report, while it also allows an effective use of time for the development of the students' competencies. The use of VBA and the working environment of Excel provides more flexibility and freedom to the professor for designing practice lectures or tests than other available platforms, in which the professor needs to adapt the structure of the exercise to pre-established forms. The development of the practice lecture in a compact Excel file facilitated the obtention of good qualifications by the students.
\end{abstract}

Keywords: problem-solving, optimization, Excel, Solver, VBA, evaluation

\section{Resumen}

El presente trabajo se centra en explotar el potencial de las herramientas VBA y Solver de Excel para la realización de un problema de optimización en una práctica de informática de la asignatura "Análisis y simulación de procesos" del grado de Ingeniería Química. Mediante el uso de macros y VBA se ha diseñado un problema en hojas de cálculo de Excel en las cuales se integran: la asignación de diferentes series de datos experimentales a los alumnos, la realización de forma guiada y estructurada de tres ejercicios, y la evaluación automatizada de las prácticas por parte del profesor. El diseño integrado y estructurado de la práctica tiene ventajas tanto para el alumnado como para el profesorado, puesto que facilita la realización y calificación de la práctica $y$ permite una utilización efectiva del tiempo para el desarrollo de competencias en los alumnos. El uso de VBA y del entorno de trabajo de Excel permite al profesor tener mayor flexibilidad y libertad para el diseño de prácticas o de exámenes que las que permiten otras plataformas existentes en 
las que el profesor tiene que adaptarse a las formas preestablecidas. El desarrollo de la práctica en un archivo compacto de Excel facilitó la obtención de buenas puntuaciones por parte de los alumnos.

Palabras clave: resolución de problemas, optimización, Excel, Solver, VBA, evaluación

\section{Introducción}

La simulación y optimización de procesos es una disciplina fundamental en el currículo de un Ingeniero Químico, ya que en el desarrollo de proyectos ingenieriles se demandan estudios de simulación con diferentes propósitos, tales como un análisis de factibilidad, un diseño conceptual o una optimización de las condiciones de operación de un proceso. En los estudios de grado de Ingeniería Química de la UPV tales conocimientos se aprenden en la asignatura "Análisis y simulación de procesos" en la que la mitad de la carga docente se dedica a la resolución de problemas.

El desarrollo informático de las últimas décadas ha propiciado un cambio en la metodología de la docencia, especialmente en la docencia universitaria, donde cada vez se exigen mayor destreza en el uso de herramientas computacionales. Las habilidades computacionales en la ingeniería química se centran de manera genérica en la utilización de hojas de cálculo y de forma específica en el uso de programas de simulación, diseño, control de procesos y programación (Kassim \& Cadbury, 1996). Las prácticas en los estudios de ingeniería química han estado profundamente influenciadas por los continuos avances en hardware y software (Wong \& Barford, 2010). Los programas utilizados para la simulación modular presentan significativas curvas de aprendizaje, donde el lenguaje algebraico suele ser desconocido por los estudiantes (Ferreira et al., 2004). Además, para la resolución de ejercicios de simulación y de optimización es necesario utilizar métodos matemáticos y de optimización, por lo que se hace uso de programas matemáticos que tengan implementados los algoritmos de cálculo necesarios. Esto permite que el alumno se centre en el objetivo global del problema y no se detenga en detalles específicos de la resolución. Con este fin, el uso de un software de cálculo sencillo y de uso habitual por los alumnos, como Excel, puede facilitar la comprensión por parte del alumno de conceptos fundamentales de la asignatura. Excel dispone de la herramienta Solver, que incluye diferentes algoritmos de optimización, lo cual permite su aplicación en prácticas informáticas de asignaturas de simulación. Existen diversas experiencias previas de utilización de hojas de cálculo en Excel y Solver en cursos de ingeniería química. S. Fasoula et al. explotaron el potencial pedagógico de las hojas de cálculo Excel para explicar la separación de solutos mediante cromatografía iónica (Fasoula, Nikitas, \& Pappa-Louisi, 2017). Otros ejemplos de la aplicación de hojas de cálculo de Excel en el campo de la ingeniería química son la resolución de problemas de química analítica (Brown, 2009) o la resolución de problemas de optimización en sistemas de intercambiadores de calor (Briones \& Escola, 2019). Por otra parte, la utilidad de las hojas de cálculo para la docencia ha sido también demostrada en otros campos, tales como las matemáticas (Ferreira 
et al., 2004), los negocios (Leong \& Cheong, 2010; Tatnall \& Reyes, 2017), o la estadística (Zaneldin \& El-Ariss, 2010).

Otra herramienta con potencial aplicación en el aprendizaje de la materia de simulación incluida en el Excel es Visual Basic for Applications (en adelante VBA). La utilización de VBA en docencia (generalmente orientada al análisis de una variable de salida a partir de una serie de variables de entrada) ha permitido mejorar las calificaciones de los alumnos y reducir los tiempos requeridos en su formación en la simulación de procesos (Court, 2004; Ferreira, Lima, \& Salcedo, 2004). Pero además el profesor puede aprovechar esta herramienta para diseñar hojas de cálculo como vía de comunicación entre el trabajo realizado por el alumno, es decir los cálculos necesarios para la resolución de un problema, y el trabajo del profesor, entendido en este contexto como la creación de datos del problema y la obtención de las respuestas de los alumnos para su evaluación.

\section{Objetivos}

El objetivo general del presente trabajo es explotar el potencial de Excel mediante el uso de las herramientas VBA y Solver para diseñar una práctica informática que facilite el desarrollo de competencias en los alumnos y facilite la labor de evaluación del profesor.

Los objetivos específicos de este trabajo son:

- Diseñar hojas de cálculo en las que el alumno puede realizar de forma organizada la resolución del ejercicio práctico

- Generar datos del enunciado del problema personalizados para cada alumno o grupo de trabajo

- Generar campos para la evaluación automática de las respuestas para un número elevado de alumnos.

\section{Desarrollo de la innovación}

En este trabajo se presenta cómo se pueden aprovechar las herramientas del Excel para diseñar una práctica informática de dos horas de duración integrada en un único archivo organizada de forma estructurada y que permite realizar la evaluación automática de los resultados obtenidos por los alumnos.

\subsection{Descripción de la práctica}

Los alumnos hacen uso de la herramienta Solver del Excel para resolver un problema de optimización. En la práctica se plantea un ejercicio de ajuste de los parámetros de un modelo, la optimización de las condiciones de operación de un proceso y el análisis de la influencia de un factor sobre el coste del proceso en cuestión. 
En el ámbito de la ingeniería química en muchos casos es necesario obtener los parámetros de los modelos mediante la minimización del error existente entre los datos experimentales y los predichos por las ecuaciones del modelo. Ejemplos típicos del ajuste de parámetros en la Ingeniería Química son el desarrollo de modelos cinéticos y la determinación de coeficientes de difusión.

La práctica está estructurada en tres apartados de complejidad creciente: mientras las restricciones y parámetros a optimizar en los dos primeros apartados son indicadas de forma detallada en el enunciado, el nivel de complejidad del segundo apartado aumenta debido al cálculo de costes de forma autónoma por el alumno y en el tercer apartado se realiza una pregunta abierta donde el alumno debe razonar de forma crítica el resultado obtenido.

En la primera parte de la práctica, los alumnos calculan los parámetros del modelo de KedemSpiegler que describe el rechazo y el flujo de permeado que pasa a través de membranas de nanofiltración. Cada grupo de alumnos recibe una serie de datos experimentales de densidad de flujo y concentración, sobre la cual definirán una función de error cuadrático. Los alumnos deberán inicializar los parámetros a calcular con un valor semilla lógico. A continuación, utilizarán la herramienta Solver de Excel para minimizar dicha función de error y obtener los parámetros del modelo. Para la resolución del ejercicio los alumnos disponen de un objeto de aprendizaje en formato vídeo de 10 minutos de duración en el que se explica el proceso de ajuste y los pasos a seguir para utilizar la herramienta Solver de Excel. El acceso libre al objeto de aprendizaje se realiza a través del repositorio de la UPV https://riunet.upv.es/ (http://hdl.handle.net/10251/63522).

En la segunda parte del ejercicio, utilizando los parámetros del modelo obtenidos anteriormente, el alumnado debe construir una función objetivo para minimizar el coste total anual de una planta de tratamiento de agua con una producción fijada, actuando sobre el área de la membrana y la presión de la bomba. Para ello se proporcionan los valores económicos y operacionales de una planta de nueva construcción y se indican una serie de restricciones a incluir durante la aplicación del comando Solver. El alumnado deberá hacer uso de los conocimientos adquiridos en otras asignaturas para crear las funciones de costes a partir de las variables del proceso.

Por último, en la tercera parte del ejercicio, los alumnos calculan y representan la influencia de la presión en el coste unitario del permeado producido sin tener en cuenta ninguna restricción. En esta parte, los alumnos deben dar una respuesta razonada del resultado obtenido.

\subsection{Diseño de la práctica}

Haciendo uso de la herramienta VBA de Excel el profesor crea el entorno de trabajo para el alumno mediante el diseño de las hojas de cálculo organizadas, con una estructura definida e instrucciones claras sobre las tareas y cálculos a realizar.

El archivo Excel diseñado para los alumnos consta de 4 hojas visibles. 
En la primera hoja se establecen los grupos de trabajo. Para esto el profesor crea varios menús desplegables donde los componentes del grupo deben buscar su nombre. Automáticamente, a cada grupo de alumnos se le asigna un caso correspondiente a una serie de datos experimentales. En total, el ejercicio cuenta con una colección de 20 series de datos experimentales en una hoja oculta, de los cuales se importa una para cada grupo de alumnos mediante una instrucción implementada en VBA. De esta forma, mediante el uso de VBA, se asegura que cada grupo realice el ajuste sobre datos diferentes, y se evita la manipulación de éstos.

Las otras tres hojas de cálculo accesibles para los alumnos contienen los tres ejercicios de los que consta la práctica descritos anteriormente. En cada una de ellas existen diferentes tipos de celdas:

- Celdas marcadas en gris que incluyen constantes no modificables por parte de los alumnos y celdas marcadas en blanco con datos experimentales del modelo asignado a cada alumno.

- Celdas asignadas a respuestas, marcadas en blanco y disponibles para que el alumno realice cálculos.

Además, cada uno de los apartados cuenta con un cuadro de texto, en el cual el alumno debe comentar los pasos seguidos y razonar de forma crítica sobre los resultados obtenidos. Cada cuadro de texto admite un máximo de 1000 caracteres, de forma que se fuerza al alumno a realizar un ejercicio de síntesis.

El profesorado puede acceder a un número mayor de hojas mediante la introducción de una clave. Una hoja de cálculo contiene el listado de alumnos y los casos asignados a cada uno de ellos. En otras dos hojas de cálculo se incluyen las 20 series de datos experimentales y los ajustes correspondientes a cada serie, respectivamente. Por último, el archivo incluye una hoja de evaluación descrita a continuación.

\subsection{Diseño del entorno de evaluación}

El uso de VBA también se empleó con el fin de agilizar y facilitar al profesor la evaluación del trabajo desempeñado por cada grupo de alumnos.

Para ello, se construye una hoja de evaluación, donde se vinculan las respuestas introducidas por los alumnos y las respuestas correctas correspondientes al caso asignado al alumno. En una columna adicional se realiza la evaluación automática del ejercicio mediante un algoritmo de comparación entre las respuestas de los alumnos y las respuestas correctas. 


\section{Resultados}

El diseño de una práctica informática con un formato organizado y claramente estructurado, donde el alumno puede identificar inmediatamente las tareas a realizar y los resultados a entregar, permite una mejor utilización del tiempo de la práctica para el desarrollo y la evaluación de diferentes competencias.

\subsection{Entorno de trabajo del alumno}

En la presente sección se muestran las diferentes hojas de cálculo creadas para la realización de la práctica.

En la Figura 1 se puede observar la hoja inicial con el menú desplegable para la creación de los grupos de trabajo. Además, la hoja presenta las instrucciones necesarias para la correcta utilización del archivo Excel por parte de los alumnos y en ella se explica el procedimiento para la correcta entrega del archivo con las respuestas finales.

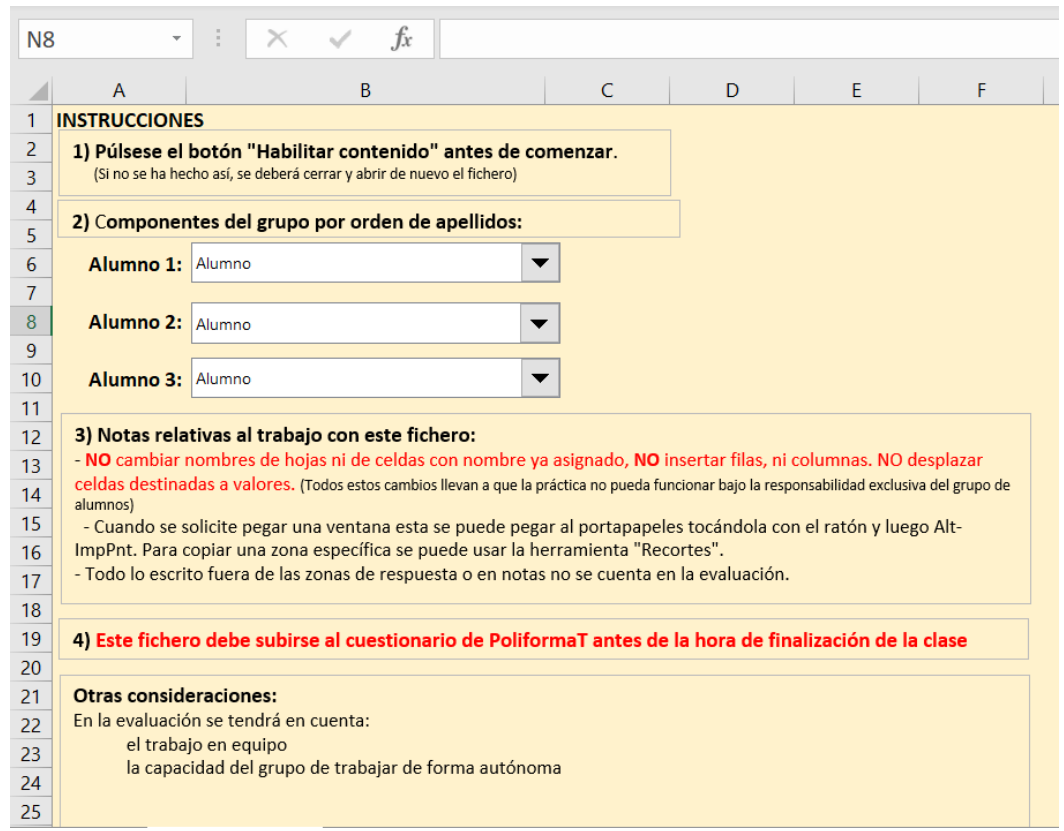

Fig. 1. Interfaz de usuario correspondiente a la primera hoja de cálculo visible por los alumnos.

En la Figura 2 se muestra la hoja de cálculo donde los alumnos realizan el primer ejercicio de la práctica. En las primeras columnas se han importado los datos experimentales asignados al alumno a partir de la hoja oculta que contiene toda la colección de datos. Como se puede observar en la figura, la hoja de cálculo tiene una estructura definida, donde el alumno puede identificar a primera vista en qué celdas debe realizar cálculos y en qué celdas se calcula la función objetivo y se ajustan los parámetros del proceso. 
La hoja de cálculo diseñada para la resolución del segundo apartado se muestra en la Figura 3. En este apartado el alumno debe rellenar las casillas indicadas con el fin de minimizar los costes totales de una planta de nueva construcción para producir $10000 \mathrm{~L} / \mathrm{h}$ de permeado modificando la presión de la bomba y el área de la membrana. Además de las casillas de cálculo, la hoja incluye una región de texto en la que el alumno debe justificar los cálculos realizados.

La hoja de cálculo correspondiente al tercer ejercicio de la práctica se muestra en la Figura 4. En este caso, la hoja cuenta no sólo con celdas dedicadas al cálculo, sino que también contiene un gráfico donde se representan los resultados obtenidos. Además, se pide a los alumnos que razonen la forma de la curva obtenida para la influencia de la presión transmembranal sobre el coste de producción de permeado.

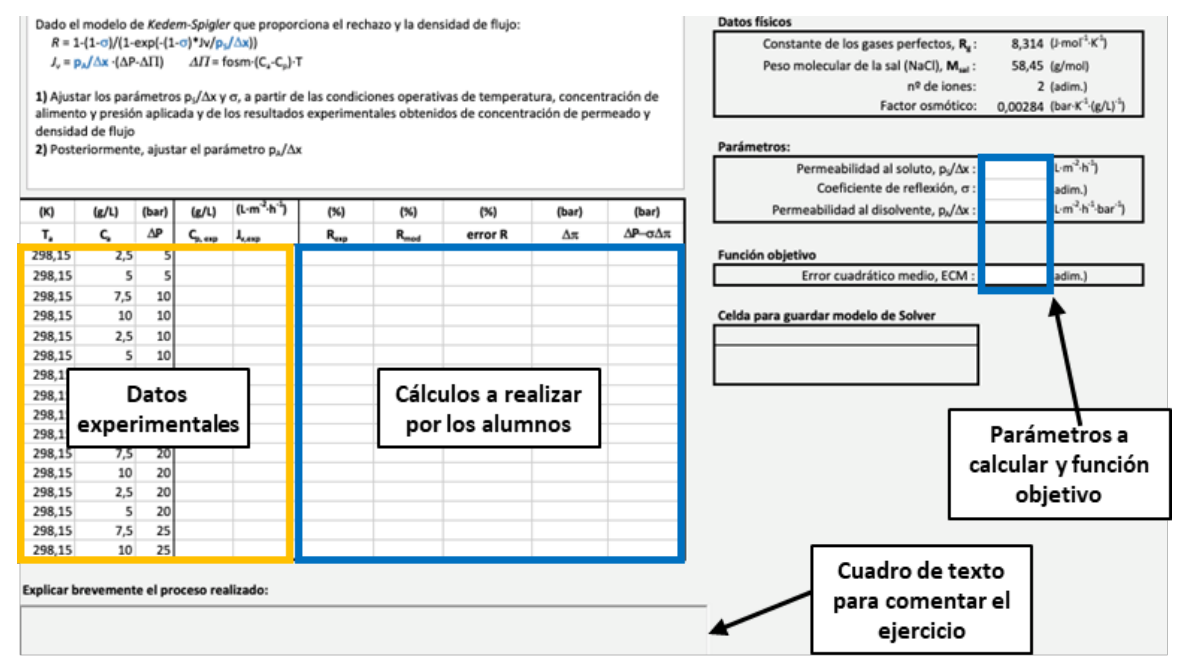

Fig. 2. Hoja de cálculo creada para la resolución de la primera parte del problema con el cálculo de los parámetros del modelo 
Uso combinado de VBA y Solver de Excel para la realización de ejercicios de optimización en ficheros Excel fácilmente evaluables

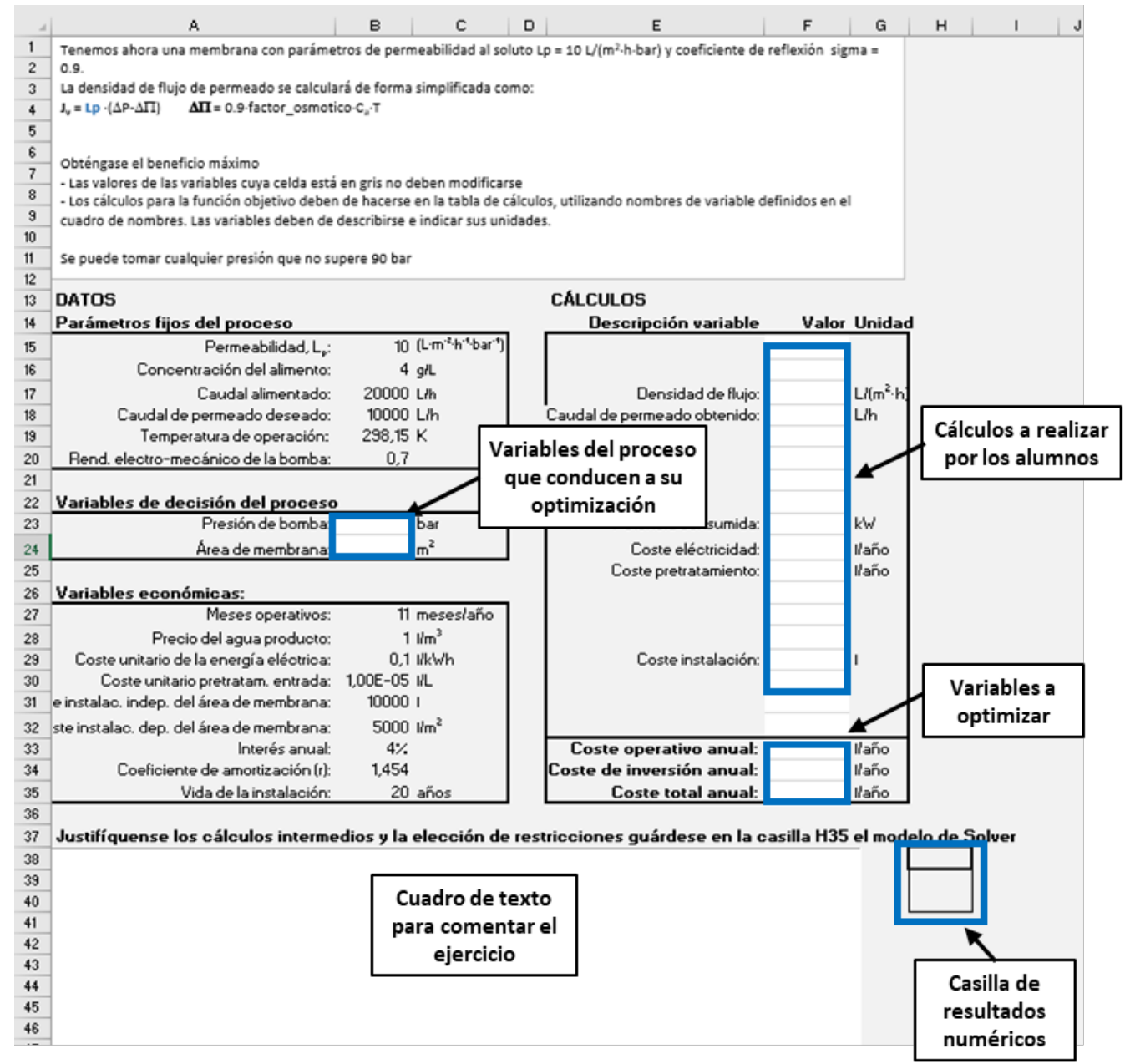

Fig. 3. Hoja de cálculo creada para la resolución de la segunda parte del problema

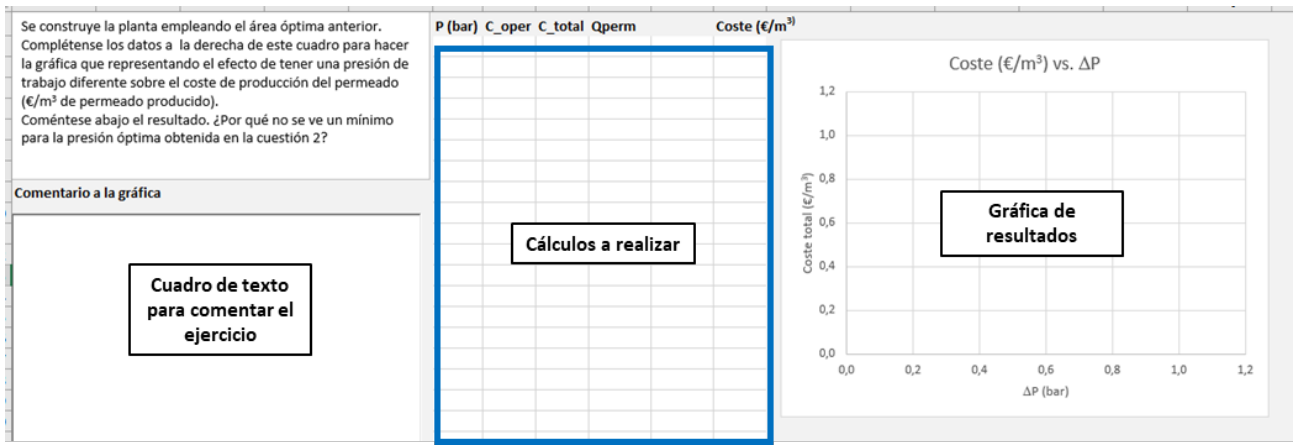

Fig. 4. Hoja de cálculo diseñada para la resolución de la tercera parte del problema 


\subsection{Entorno de trabajo del profesor}

La inclusión de los datos de los alumnos y la asignación directa mediante un comando de VBA de las series de datos experimentales es una herramienta de automatización que facilita la corrección del profesor.

La hoja de evaluación (Fig. 5) permite la rápida evaluación de un número elevado de alumnos mediante la introducción de un botón diseñado para activar la comparación lógica entre los resultados obtenidos y la solución correcta. Frente a otras herramientas para la creación y evaluación de exámenes (p.ej. la plataforma PoliformaT utilizada en la UPV), esta herramienta presenta la ventaja adicional de ser adaptable a diferentes tipos de prácticas por parte del profesor.

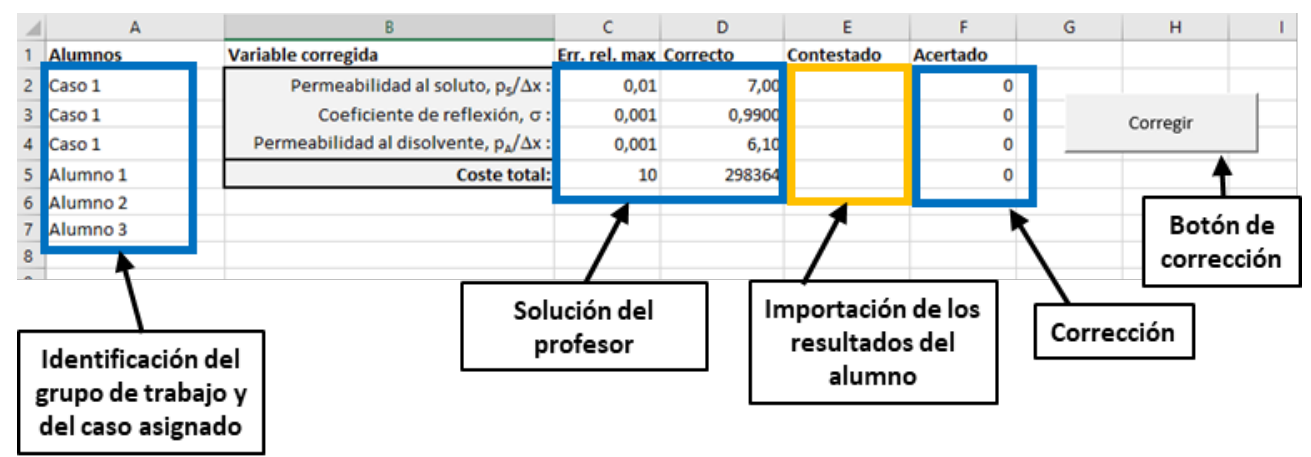

Fig. 5. Vista de la hoja de cálculo dedicada a la evaluación del profesor.

\subsection{Desarrollo de competencias por parte de los alumnos}

Mediante la práctica diseñada los alumnos trabajan las siguientes competencias:

- Competencia específica 16(ES): Capacidad para el análisis, diseño, simulación y optimización de procesos y productos: En el primer y segundo apartado, los alumnos realizan un ejercicio de optimización donde deben definir una función objetivo a minimizar. Además, en el tercer ejercicio de la práctica, los alumnos realizan el análisis de la influencia de un parámetro de operación del proceso estudiado.

- Competencia transversal CT13: Instrumental específica entendida aquí como el uso de la herramienta Solver de Excel.

- Competencia transversal CT2: Aplicación y pensamiento práctico: Puesto que el alumno debe trasladar las indicaciones del objeto de aprendizaje utilizado para la resolución del primer apartado a la resolución del segundo.

- La competencia transversal CT3: Análisis y resolución de problemas: El alumno debe vincular las funciones de optimización y las restricciones con las variables del proceso.

- Competencia transversal CT8: Comunicación efectiva: la inclusión de cuadros de texto para la justificación de los resultados con un número limitado de caracteres 
también potencia esta competencia dado que el alumno debe argumentar de forma concisa y eficaz adaptándose al espacio proporcionado para ello.

\subsection{Evaluación de los resultados}

A continuación, se muestran los resultados obtenidos por los alumnos en el curso 2015/16. La gran mayoría ( $>50 \%$ de los alumnos) obtuvo calificaciones en la práctica por encima del 9 (Fig. 6). También se puede observar que todos los alumnos obtuvieron una calificación superior al 7.

Fig. 6. Notas finales del alumnado para la práctica informática.

Respecto al grado de dificultad de cada apartado, en la Fig. 7 se desglosa la distribución de notas por ejercicios, considerándose una puntuación normalizada en base 1. En términos generales, puede observarse que el número de personas que consigue sacar la máxima nota decrece a medida que se otorga mayor libertad de acción al alumno. En la primera parte, que es la más cerrada y detallada, un $94,37 \%$ de los alumnos logran obtener la máxima nota. En la segunda parte, donde se requiere consultar y tener en cuenta ecuaciones de potencias y costes, la distribución de notas es más homogénea, con un porcentaje de alumnos que consiguen la máxima nota de un 30,99\% (Fig. 7). Finalmente, la tercera parte, en la cual se requiere de una explicación fundamentada en los conceptos del análisis y simulación, la mayoría de los alumnos ( $>75 \%$ ) obtiene sólo un cuarto de la nota total, cayendo el porcentaje de alumnos que consiguen la máxima nota hasta el 5,63\%. En general, se puede concluir que la distribución de notas de los alumnos se desplaza hacia valores más bajos a medida que el número de operaciones a realizar y el nivel de análisis crítico aumenta. 


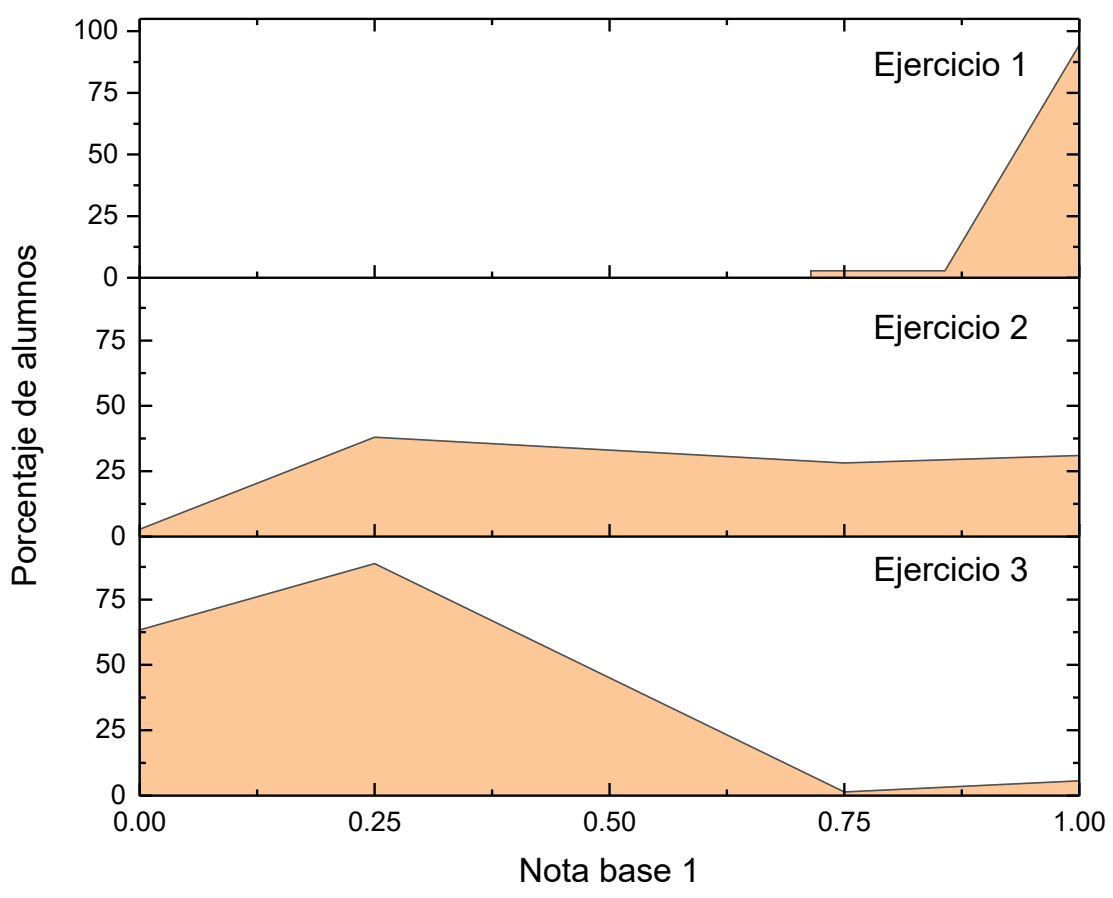

Fig. 7. Distribución de notas obtenidas por los alumnos en los tres ejercicios de la práctica.

Además de los datos recabados durante el curso 2015/16, la realización de los ejercicios 1 y 2 se propuso a los alumnos del curso 2018/19 como tarea voluntaria con el fin de evaluar el nivel de desarrollo de la competencia "CT11 - Aprendizaje permanente". El primer dato significativo es el nivel de participación alcanzado, dado que un 90\% de los alumnos (63 de un total de 70 matriculados) visualizó el vídeo y respondió un cuestionario acerca de éste; y un $75.7 \%$ (53 de 70) realizó el ejercicio propuesto y envió sus respuestas a través de la plataforma PoliformaT.

En el cuestionario acerca del vídeo se preguntó sobre los conceptos nuevos aprendidos mediante su visualización, así como sobre el nivel de refuerzo de los conceptos tratados anteriormente en la asignatura. Los resultados de dicho cuestionario se resumen en la Fig. 8. Un 79.4\% de los alumnos aprendieron la utilidad de asignar nombres de variables a celdas y rangos de datos, mientras que un $20.6 \%$ tenían conocimiento previo acerca de dicha función. Por el contrario, una amplia mayoría había utilizado la función Solver de Excel en cursos previos. Asimismo, el 95.2\% de los alumnos que visualizaron el video consideran que éste les ayudó a reforzar conceptos de optimización impartidos en la asignatura Análisis y simulación de procesos. 
a) Asignación de nombres de variables a celdas

b) Refuerzo del manejode Solver

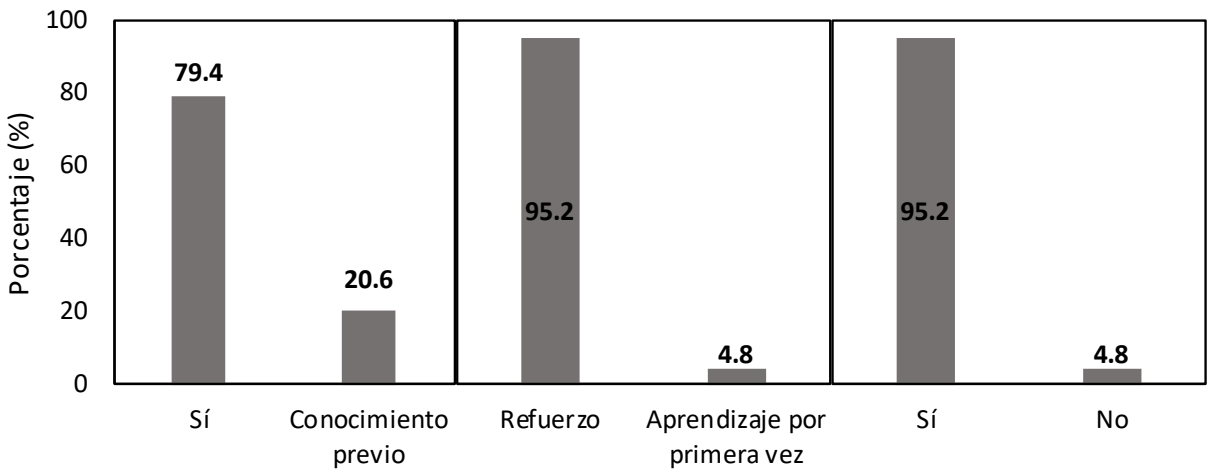

Fig. 8. Respuestas de los alumnos al cuestionario realizado acerca de los conocimientos aprendidos mediante la visualización del vídeo explicativo sobre la utilización de la herramienta Solver de Excel.

Tras la realización del ejercicio, los alumnos contestaron un cuestionario adicional, cuyos resultados más significativos se presentan en la Fig. 9. Por lo que respecta a la opinión del alumnado sobre la realización y tipología de actividad, más de un 50\% le otorga una puntuación igual o mayor a 7 al método de aprendizaje utilizado. Dado que los alumnos trabajaron de forma autónoma desde casa, también se les consultó acerca del tiempo que necesitaron para realizar los ejercicios. Los resultados (Fig. 9 b)) indican que el tiempo de realización de la tarea fue menor de 2 horas en la mayoría de los casos. Teniendo en cuenta que en el curso 2018/19 no se realizó el tercer ejercicio, estos resultados indican que el tiempo requerido para la actividad es similar al de una clase práctica en presencia del profesorado. Por lo tanto, se puede confirmar que tanto el conocimiento previo de Excel como la visualización del vídeo explicativo permiten a los alumnos aprender en un tiempo razonable trabajando de forma autónoma desde casa. 
a)

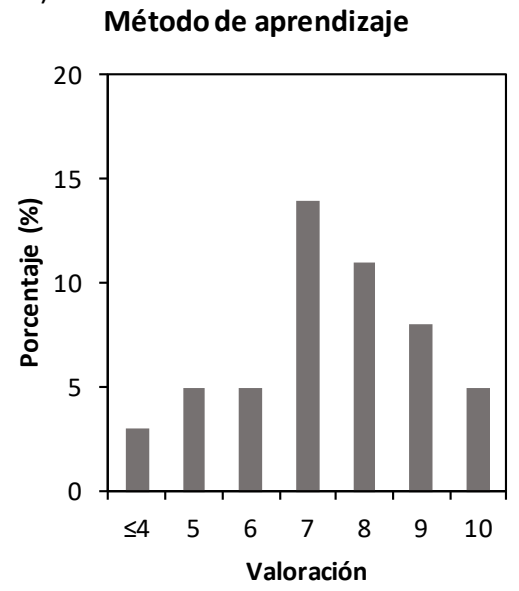

b)

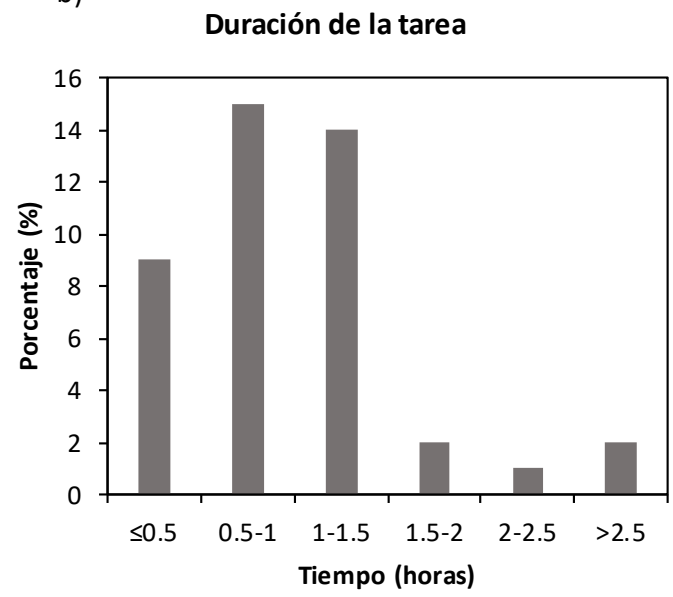

Fig. 9. (a) Valoración de 0 a 10 por parte de los alumnos del método de aprendizaje y (b) tiempo requerido por los alumnos para la realización de la tarea.

\section{Conclusiones}

En el presente trabajo se han estudiado las ventajas que conlleva la realización de una práctica informática integrada en un único archivo Excel, diseñado y estructurado mediante el uso de VBA, y en la cual los alumnos han de resolver un problema de optimización utilizando la herramienta Solver.

La utilización de Solver como herramienta de optimización, permite resolver un problema típico en ingeniería química como es el ajuste de los parámetros de un modelo. La principal ventaja del uso de Excel es que, al tratarse de un entorno de cálculo previamente conocido por los alumnos, éstos tienen menos dificultades relacionadas con el uso del software de cálculo, y se puede dedicar más tiempo a trabajar los conceptos de la asignatura. La herramienta VBA permite la inclusión de cuadros de texto con un número limitado de caracteres, donde los alumnos deben razonar y discutir los resultados obtenidos de forma concisa.

Mediante el uso de VBA, el profesor puede realizar un diseño compacto de la práctica en un único archivo de Excel, en el cual se integran los nombres de los alumnos vinculados a diferentes datos experimentales, los ejercicios a realizar, y una hoja de evaluación con el cálculo de las notas obtenidas por los alumnos. Esta hoja de calificación es útil para el profesorado, ya que permite una evaluación más rápida de un número muy elevado de alumnos. Mediante el uso de esta herramienta y del entorno de trabajo de Excel el profesor tiene mayor flexibilidad y libertad para el diseño de prácticas o de exámenes que las que permiten otras plataformas existentes en las que el profesor tiene que adaptarse a las formas preestablecidas (como es el caso del uso de PoliformaT en la UPV).

La utilización de la práctica diseñada en el curso 15/16 puso de manifiesto que la organización y estructuración del entorno de cálculo permitió que un elevado número de 
alumnos completara la práctica con éxito. No obstante, a medida que el número de cálculos a realizar y el grado de análisis crítico demandado en los ejercicios aumenta, decrece el porcentaje de alumnos que obtienen una puntuación elevada.

La realización de parte de los ejercicios de la práctica se propuso como tarea voluntaria a realizar en casa a los alumnos del curso 18/19. En general, la metodología propuesta fue bien recibida por parte del alumnado, obteniéndose niveles de participación elevados. Los alumnos valoran positivamente la metodología empleada. Cabe destacar, que el tiempo requerido para la realización de la actividad es similar al tiempo de una práctica informática de la asignatura, confirmándose que la metodología empleada es aplicable tanto durante la realización de prácticas informáticas monitorizadas por el profesor, como en forma de tarea voluntaria a realizar en casa por parte de los alumnos.

\section{Referencias}

BRIONES, L., \& ESCOLA, J. M. (2019). "Application of the Microsoft Excel Solver tool in the solution of optimization problems of heat exchanger network systems". Education for Chemical Engineers, 26, 41-47.

BROWN, P. (2009). "Understanding Solubility through Excel Spreadsheets". Journal of Chemical Education, 78(2), 268. https://doi.org/10.1021/ed078p268

COURT, M. C. (2004). "The Impact of Using Excel Macros for Teaching Simulation Input and Output Analysis". International Journal of Engineering Education, 20(6), 966-973.

FASOULA, S., NIKITAS, P., \& PAPPA-LOUISI, A. (2017). "Teaching Simulation and ComputerAided Separation Optimization in Liquid Chromatography by Means of Illustrative Microsoft Excel Spreadsheets". Journal of Chemical Education, 94(8), 1167-1173. https://doi.org/10.1021/acs.jchemed.7b00108

FERREIRA, E. C., LIMA, R., \& SALCEDO, R. (2004). "Spreadsheets in Chemical Engineering education - A tool in process design and process integration". International Journal of Engineering Education, 20(6), 928-938.

KASSIM, H. O., \& CADBURY, R. G. (1996). "Pergamon The Place of the Computer in Chemical Engineering Education" H . O . Kassim and R . G . Cadbury, Division of Chemical Engineering, South Bank University, Borough Road, LONDON SE1 0AA. Computers Chemical Engineering, 20, 13411346.

LEONG, T.-Y., \& CHEONG, M. L. F. (2010). "Teaching Business Modeling Using Spreadsheets". INFORMS Transactions on Education, 9(1), 20-34. https://doi.org/10.1287/ited.1080.0015

TATNALL, A., \& REYES, G. (2017). "Teaching IT Project Management to Postgraduate Business Students: A Practical Approach". Journal of Information Technology Education: Research, 4, 153166. https://doi.org/10.28945/270

WONG, K. W. W., \& BARFORD, J. P. (2010). "Teaching Excel VBA as a problem solving tool for chemical engineering core courses". Education for Chemical Engineers, 5(4), e72-e77. https://doi.org/10.1016/j.ece.2010.07.002

ZANELDIN, E., \& EL-ARISS, B. (2010). "Using spreadsheets and VBA for teaching civil engineering concepts”. ICSIT 2010 - International Conference on Society and Information Technologies, Proceedings, 445-450. 